\title{
Hybrid Pilot/Quantization based Feedback in Multi-Antenna TDD Systems
}

\author{
Umer Salim*, David Gesbert*, Dirk Slock* and Zafer Beyaztas ${ }^{\dagger}$ \\ ${ }^{*}$ Mobile Communications Department \\ Eurecom, France \\ Email: \{salim, gesbert, slock\}@eurecom.fr \\ †Accenture, Gustav Mahlerplein 90, 1082 MA, Amsterdam \\ Email: zafer.beyaztas@accenture.com
}

\begin{abstract}
The communication between a multiple-antenna transmitter and multiple receivers (users) with either a single or multiple-antenna each can be significantly enhanced by providing the channel state information at the transmitter (CSIT) of the users, as this allows for scheduling, beamforming and multiuser multiplexing gains. The traditional view on how to enable CSIT has been as follows so far: In time-division duplexed (TDD) systems, uplink (UL) and downlink (DL) channel reciprocity allows for the use of a training sequence in any given uplink slot, which is exploited to obtain an uplink channel estimate. This estimate is in turn recycled in the next downlink slot. In frequency-division duplexed (FDD) systems, which lack the UL and DL reciprocity, the CSIT is provided via the use of a dedicated feedback link of limited capacity between the receivers and the transmitter. In this paper, we focus on TDD systems and show that the traditional TDD CSIT acquisition fails to fully exploit the channel reciprocity in its true sense. In fact, we show that the system can benefit from a combined CSIT acquisition strategy mixing the use of limited feedback and that of a training sequence. We demonstrate the potential of our approach in terms of improved CSIT quality under a global training and feedback resource constraint.
\end{abstract}

\section{INTRODUCTION}

Multiple-antenna transmitters and receivers are instrumental to optimizing the performance of bandwidth and power limited wireless communication systems [1], [2]. In the downlink (DL), in particular, the communication between a multipleantenna enabled base station (BS) and one or more users with either a single or multiple antenna each can be significantly enhanced through the use of scheduling, beamforming and power allocation algorithms, be it in single user or multi-user mode. To allow for beamforming and/or multiuser multiplexing capability, the BS transmitter must however be informed with the channel state information of each of the served users [3], [4], [5], except when the number of users reaches an asymptotic (large) regime in which case random opportunistic beamforming scheme can be exploited [6], [7]. This has motivated the proposal of many techniques for providing the channel state information at the transmitter (CSIT) in an efficient manner. Proposals for how to provide CSIT roughly fall in two categories depending upon the chosen duplexing scheme for the considered wireless network. In the case of time-division duplex (TDD) systems, it was proposed to exploit the reciprocity of the uplink (UL) and DL channels, so as to avoid the use of any feedback channel [8], [9], [10]. The way reciprocity is exploited, in the current TDD systems, is through the use of a training sequence sent by the user on the UL, based on which the BS first builds an estimate of the UL channel which in turn serves as an estimate for the DL channel in the next DL slot [8]. In frequency-division duplex (FDD) scenarios, the lack of channel reciprocity motivates instead the use of a dedicated feedback link in which the user conveys the information about the estimated DL channel, back to the BS. Recently, several interesting strategies have been proposed for how to best use a limited feedback channel and still provide the BS with exploitable CSIT [11], [12], [13].

Although in the past, the balance has weighed in the favor of FDD systems when choosing a duplexing scheme (in part because of heavy legacy issues in voice oriented $2 \mathrm{G}$ networks and also because of interference management between UL and DL), current discussions in the standardization groups indicate an increasing level of interest for TDD for upcoming wireless data-access networks (e.g.WiMax, etc.), caused partly by its advantages in maintaining system flexibility with respect to UL and DL traffic loads, and mostly because TDD systems are seen as more efficient in providing the CSIT required by several MIMO DL schemes, thanks to the channel reciprocity.

In this paper, we focus on the problem of CSIT acquisition in a TDD system. We take a step back and shed some critical light on the traditional approach above consisting in exploiting the channel reciprocity via the use of training sequences exclusively. The key idea is as follows: when sending a training sequence in the UL of a traditional TDD system, the user allows the BS to estimate the channel by a classical channel estimator (it can be a least-square (LS) estimator or minimum mean square error (MMSE) based, just to name a few). However, note that the user itself has the knowledge of the channel coefficients (obtained during the UL frame or from the DL synchronization sequence or other control signals or even from the previous DL frame if channel is correlated in time) but, regretfully, does not exploit that knowledge in order to facilitate the CSIT acquisition by the BS. Instead, it uses this knowledge only for its own detection purposes.

Interestingly, by contrast, in FDD systems, the user exploits its channel knowledge by quantizing the channel and sending the result over a dedicated feedback link. In this case, UL 
training is used by the BS solely for UL data detection as this UL training cannot give any direct information to the BS about the DL channel coefficients. Actually in FDD systems, UL and DL portions of the bandwidth are normally selected quite apart and hence channel realizations over respective bandwidths can be safely assumed to be independent of each other, so there is no channel reciprocity in FDD systems.

We propose that, while dealing with TDD systems, there is a clear opportunity to combine both forms of CSIT acquisition strategies. As a result, we investigate a scheme mixing classical channel estimation using training with limited feedback carrying information about the quantized channel. This gives us a framework for fully utilizing the channel reciprocity in a TDD setup and it improves the classical trade-off between CSIT estimation accuracy and training/feedback resource usage. We characterize the optimal CSIT acquisition structure and then propose a sub-optimal outage rate based approach which helps us to optimize the fixed resource partitioning between training and quantized feedback phases. The results obtained confirm our intuition and demonstrate the potential of this hybrid (mix of training and quantized feedback) approach for upcoming TDD systems.

The paper is structured as follows: System model is given in section II, followed by classical CSIT acquisition in TDD and FDD systems in section III. Optimal training and feedback combining strategy is explained in section IV with outage based optimization framework in section V. Simulation results appear in section VI followed by conclusions in section VII. Notation: $\mathbb{E}$ denotes statistical expectation. Lowercase letters represent scalars, boldface lowercase letters represent vectors, and boldface uppercase letters denote matrices. $\mathbf{A}^{\mathbf{T}}, \mathbf{A}^{\dagger}, \mathbf{A}^{-\mathbf{1}}$, $\mathbf{A}^{+}$denote the transpose, the Hermitian, the inverse and the pseudo-inverse of matrix $\mathbf{A}$, respectively. For a vector $\mathbf{a},\|\mathbf{a}\|$ and $\overline{\mathbf{a}}$ represent, respectively, its norm and unit-norm direction vector so that $\mathbf{a}=\|\mathbf{a}\| \overline{\mathbf{a}}$.

\section{SySTEM Model AND CSIR ACQUisition}

We consider the two way communication in a cell between a single BS, equipped with $M$ antennas, and a single antenna mobile user. The channel $\mathbf{h} \in \mathcal{C}^{M}$ is assumed to be flatfading with independent complex Gaussian zero-mean unitvariance entries, where $\mathcal{C}^{M}$ represents the $M$-dimensional complex space. We assume block fading channel so each channel realization stays constant for $T$ symbol intervals [14] which can be accordingly partitioned between UL and DL data transmissions. Extensions to $\operatorname{OFDM}(\mathrm{A})$ and multi-user systems are possible and straightforward.

The goal of this paper is to provide a reliable estimate of the DL channel to the BS, which in turn can be used for beamforming/precoding purposes. However we focus on the acquisition issue of the channel knowledge and not about its use in MIMO transmission schemes.

In the downlink, the received signal at the user for $L$ symbol intervals is given by

$$
\mathbf{y}_{\mathrm{dl}}=\mathbf{X}_{\mathrm{dl} \mathbf{l}} \mathbf{h}+\mathbf{n}_{\mathrm{dl}},
$$

where $\mathbf{X}_{\mathbf{d l}} \in \mathcal{C}^{L \times M}$ is the signal transmitted by the BS during $L$ symbol intervals (satisfying BS power constraint), $\mathbf{n}_{\mathrm{dl}} \in \mathcal{C}^{L}$ is the complex Gaussian noise with independent zero-mean unit-variance entries and $\mathbf{y}_{\mathbf{d l}} \in \mathcal{C}^{L}$ is the observation sequence during this $L$-length interval.

If we want to use the above DL system equation for channel estimation, for identifiability of $M$-dimensional channel at the user's side, the length of the transmitted data (training sequence) should be larger than $M$. Based upon the knowledge of the transmitted data $\mathbf{X}_{\mathbf{d l}}$ (the training sequence) and the observed sequence $\mathbf{y}_{\mathbf{d l}}$, the user can estimate the DL channel h. The LS estimate would be

$$
\check{\mathbf{h}}_{\mathbf{L S}}=\mathbf{X}_{\mathrm{dl} l}^{+} \mathbf{Y}_{\mathrm{dl}} \text {. }
$$

The user can make a better channel estimate using MMSE criteria, and the estimate is given by

$$
\check{\mathbf{h}}_{\mathrm{MMSE}}=\mathbf{X}_{\mathrm{dl}}^{\dagger}\left(\mathbf{X}_{\mathrm{dl}} \mathbf{X}_{\mathrm{dl}}^{\dagger}+\mathbf{I}_{\mathbf{L}}\right)^{-1} \mathbf{y}_{\mathrm{dl}} \text {. }
$$

In the above equation, $\mathbf{I}_{\mathbf{L}}$ is the identity matrix of $L$ dimensions.

\section{Classical CSIT ACQuisition IN FDD AND TDD}

We briefly review the classical approaches for acquiring CSIT at the BS in FDD and TDD systems.

\section{A. FDD Systems}

In FDD systems, the mobile station first obtains the DL channel estimate $\check{\mathbf{h}}$ as described in the previous section. Then it needs to quantize the channel using some pre-selected codebook. If $Q$ denotes the quantization function, then for the DL channel estimate $\check{\mathbf{h}}$, its quantized version (which is actually the index of the closest channel code in the codebook) is given by $Q(\check{\mathbf{h}})$. Afterwards user maps this index (sequence of bits) into a sequence of constellation symbols, using the mapping function denoted by $S$. Hence the feedback of the DL channel would be

$$
\mathbf{x}_{\mathbf{q}}=S(Q(\check{\mathbf{h}}))
$$

where $\mathbf{x}_{\mathbf{q}} \in \mathcal{C}^{1 \times T_{q}}$ is the $T_{q}$ dimensional row vector of normalized constellation symbols representing the information of the quantized channel. Due to independent channel realizations over UL and DL of FDD systems, BS first needs to know/estimate the UL channel $\left(\mathbf{h}_{\mathbf{u}} \in \mathcal{C}^{M}\right)$ to decode the feedback $\left(\mathbf{x}_{\mathbf{q}}\right)$ properly which will be sent as UL payload. If $\mathbf{x}_{\mathbf{a}} \in \mathcal{C}^{1 \times T_{a}}$ denotes the normalized training sequence of length $T_{a}$ in the UL direction, the signal received at the BS for $T_{a}$ symbol intervals is given by

$$
\mathbf{Y}_{\mathbf{a}}=\sqrt{P} \mathbf{h}_{\mathbf{u}} \mathbf{x}_{\mathbf{a}}+\mathbf{N}_{\mathbf{a}}
$$

where $\mathbf{N}_{\mathbf{a}} \in \mathcal{C}^{M \times T_{a}}$ represents the spatio-temporally white Gaussian noise and $\mathbf{Y}_{\mathbf{a}} \in \mathcal{C}^{M \times T_{a}}$ is the received signal at $M$ antennas of the BS during this $T_{a}$-length training interval. $P$ represents the user's peak power constraint. After observing $\mathbf{Y}_{\mathbf{a}}$, the BS can make an estimate $\hat{\mathbf{h}}_{\mathbf{u}}$ of the UL channel $\mathbf{h}_{\mathbf{u}}$, knowing the training sequence $\mathbf{x}_{\mathbf{a}}$. 
Now if user transmits the quantized channel feedback $\mathbf{x}_{\mathbf{q}}$ on the UL, BS can decode this information, having an estimate $\hat{\mathbf{h}}_{\mathbf{u}}$ of the UL channel $\mathbf{h}_{\mathbf{u}}$.

$$
\mathbf{Y}_{\mathbf{q}}=\sqrt{P} \mathbf{h}_{\mathbf{u}} \mathbf{x}_{\mathbf{q}}+\mathbf{N}_{\mathbf{q}}
$$

where $\mathbf{Y}_{\mathbf{q}}$ and $\mathbf{N}_{\mathbf{q}}$ are $M \times T_{q}$ matrices of the received signal and the noise respectively at $M$ antennas of the BS during this explicit $T_{q}$ length feedback interval. So based upon the estimate $\hat{\mathbf{h}}_{\mathbf{u}}$ and the received feedback $\mathbf{Y}_{\mathbf{q}}$, BS tries to recover the DL channel feedback (quantized version, $\mathbf{x}_{\mathbf{q}}$ ) using the optimum (although relatively complex) maximum likelihood sequence estimation technique.

$$
\hat{\mathbf{h}}=\underset{\check{\mathbf{h}}}{\arg \min }\left\|\mathbf{Y}_{\mathbf{q}}-\sqrt{P} \hat{\mathbf{h}}_{\mathbf{u}} S(Q(\check{\mathbf{h}}))\right\|^{2}
$$

Actually the search space will be restricted to the codebook, hence the BS, at best, can estimate the quantized version of the channel.

\section{B. TDD Systems}

If the communication system is operating under TDD mode, DL and UL channels are reciprocal, hence $\mathbf{h}_{\mathbf{u}}=\mathbf{h}$. So if a user transmits pilot sequence on the UL (like eq. (5)), the simple (UL) channel estimation at the BS furnishes CSIT due to UL and DL channel reciprocity. In the past, this has been the classical way of getting CSIT in TDD systems [8], [9].

\section{Optimal Training and Feedback Combining in TDD SYSTEMS}

We propose a novel hybrid two stage CSIT acquisition strategy which exploits the channel reciprocity and user's channel knowledge at the same time (which is ignored in classical acquisition). We assume perfect channel knowledge at user's side for ease of exposition. Working under a constraint of fixed resource available for CSIT acquisition $\left(T_{f b}\right.$ symbol intervals and user's power constraint of $P$ ), our strategy consists of dividing this interval in two phases. A typical DL frame with this hybrid CSIT acquisition strategy has been shown in Fig. (1).

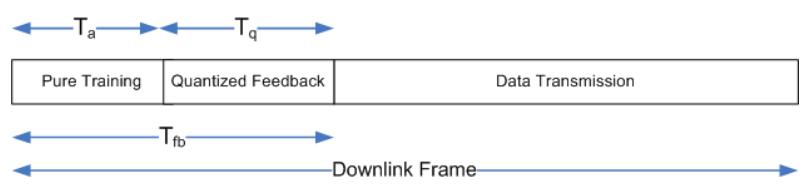

Fig. 1. Downlink frame structure with Novel Hybrid CSIT Acquisition Approach: Total feedback length is divided between pure training and quantized feedback phases.

The first stage of this hybrid approach, termed as "pure training", is the transmission of training sequence from the user to the BS for $T_{a}$ symbol intervals and the received signal will be

$$
\mathbf{Y}_{\mathbf{a}}=\sqrt{P} \mathbf{h} \mathbf{x}_{\mathbf{a}}+\mathbf{N}_{\mathbf{a}}
$$

(See eq. (5) for the dimensions of all parameters.)

The optimal training based estimate based upon the observed signal $\mathbf{Y}_{\mathbf{a}}$, knowing $\mathbf{x}_{\mathbf{a}}$, will be

$$
\hat{\mathbf{h}}_{\mathbf{a}}=\underset{\mathbf{h}}{\arg \min }\left\|\mathbf{Y}_{\mathbf{a}}-\sqrt{P} \mathbf{h x}_{\mathbf{a}}\right\|^{2}
$$

The second stage, termed as "quantized feedback", consists of the transmission of digitally quantized channel, already known at the user, for $T_{q}$ symbol intervals and the received signal will be

$$
\mathbf{Y}_{\mathbf{q}}=\sqrt{P} \mathbf{h} \mathbf{x}_{\mathbf{q}}+\mathbf{N}_{\mathbf{q}}
$$

(See eq. (6) for the dimensions of all parameters.)

where $\mathbf{x}_{\mathbf{q}}=S(Q(\mathbf{h}))$. This equation reveals the intriguing aspect that BS needs to acquire $\mathbf{h}$ which appears both as channel and data $\mathbf{x}_{\mathbf{q}}$. BS can try to decode the feedback based upon the knowledge of $\hat{\mathbf{h}}_{\mathbf{a}}$ (obtained as in eq. (9) making use of pure training $\mathbf{x}_{\mathbf{a}}$ )

$$
\hat{\mathbf{h}}_{\mathbf{q}}=\underset{\mathbf{x}_{\mathbf{q}}}{\arg \min }\left\|\mathbf{Y}_{\mathbf{q}}-\sqrt{P} \hat{\mathbf{h}}_{\mathbf{a}} \mathbf{x}_{\mathbf{q}}\right\|^{2} .
$$

The optimal CSIT will be obtained by the joint estimation and detection ( of $\mathbf{h}$ and $\mathbf{x}_{\mathbf{q}}$ ) based upon the observation of $\mathbf{Y}_{\mathbf{a}}$ and $\mathbf{Y}_{\mathbf{q}}$, knowing $\mathbf{x}_{\mathbf{a}}$ and involving an optimal split between the training and the quantized feedback (constrained as $T_{a}+$ $\left.T_{q}=T_{f b}\right)$.

$$
\hat{\mathbf{h}}=\underset{\mathbf{h}, T_{a}}{\arg \min }\left\|\left[\mathbf{Y}_{\mathbf{a}} \mathbf{Y}_{\mathbf{q}}\right]-\sqrt{P} \mathbf{h}\left[\mathbf{x}_{\mathbf{a}} S(Q(\mathbf{h}))\right]\right\|^{2}
$$

The optimal solution requires a double minimization and does not seem to bear a closed form expression for $\hat{\mathbf{h}}$. Therefore accepting some sub-optimalities, we give a framework in the next section, based upon the idea of outage rate, which allows us to obtain a simplified solution for the hybrid approach.

\section{Outage Based Training And FeEdBack PARTITIONING}

\section{A. Definitions and Initial Setup}

In this section, we give a strategy, under some assumptions, which allows optimizing the partitioning of the feedback resource between the training and the quantized feedback.

For the quantized feedback part, we employ vector quantization rather than scalar quantization of individual channel coefficients. We restrict the user to quantize the unit-norm channel direction information (CDI) vector using random vector quantization (RVQ) and feed it back. For RVQ very nice closed form expressions for the quantization error are known [12], [15]. So our analysis assumes the use of RVQ codebooks for CDI quantization. In practice, another complementary scalar should also be fed back from the users to the BS giving the link quality information such as SINR [13].

The solution for the optimal CSIT estimate, $\hat{\mathbf{h}}$ in eq. (12), requires joint estimation and detection along with an optimization over the resource split. Even if, as a simplification, we focus separately on training based estimate $\hat{\mathbf{h}}_{\mathbf{a}}$ (eq. (9)) and digital feedback based estimate $\hat{\mathbf{h}}_{\mathbf{q}}$ (eq. (11)), two questions arise: i) how the CSIT acquisition interval $T_{f b}$ should be split 
between training and feedback?, and ii) how the two estimates should be combined to get the final estimate?

Embracing some sub-optimalities, we propose the use of quantized estimate $\hat{\mathbf{h}}_{\mathbf{q}}$ as the final CSIT estimate $\hat{\mathbf{h}}$. So the quantized feedback $\mathbf{x}_{\mathbf{q}}$, which provides $\hat{\mathbf{h}}_{\mathbf{q}}$, is decoded based upon the training based estimate $\hat{\mathbf{h}}_{\mathbf{a}}$. This settles the 2 nd question above.

The choice of selecting the quantized feedback as the final CSIT estimate makes it clear that the quantization error will always sneak in the final estimate. Apart from that, the incorrect detection of digital feedback will wreak havoc on the final CSIT estimate. As the channel stays constant for each feedback interval, we are dealing with slow fading channels for which deep channel fades (causing outage) are the typical error events [16]. We propose to use the minimization of the mean square error (MSE) of CSIT, based upon the outage rate, as the criterion for the optimal split, thus answering the first question above for which we give the detailed framework in the next subsection.

This two stage outage based strategy consists of first providing a training based estimate to the BS in the training interval of length $T_{a}$. In the second interval of length $T_{q}$, user sends the quantized version (using RVQ) of its unit-norm CDI. We suppose that channel statistics are such that the user can send $b$ bits per symbol interval with an outage probability of $\epsilon$. So $b$ is the $\epsilon$-outage rate of the UL channel (see [16] for details). That means the user can send a total of $B=b T_{q}$ feedback bits at $\epsilon$ outage. Now the user should map $B$ bits over $T_{q}$ constellation symbols to transmit on the UL channel. Although the constellations used in practice have $2^{b}$ points where $b$ must be a positive integer, we relax this restriction and allow positive real values for $b$.

We define the CDI error as the sin of the angle $(\theta)$ between the true channel direction vector $\overline{\mathbf{h}}$ and the BS estimated direction vector $\overline{\hat{\mathbf{h}}}$, denoted as $\sigma^{2}(\mathbf{h}, \hat{\mathbf{h}})$.

$$
\sigma^{2}(\mathbf{h}, \hat{\mathbf{h}}) \triangleq \sin ^{2}(\theta)=1-\cos ^{2}(\theta)=1-\left|\overline{\mathbf{h}}^{\dagger} \overline{\hat{\mathbf{h}}}\right|^{2}
$$

When the channel is not in outage and the BS is able to decode the feedback correctly, there is only quantization error. On the other hand, when the channel is in outage (happens with probability $\epsilon$ ), BS cannot decode the feedback information. Hence MSE of CSIT $\sigma^{2}$ can be written as

$$
\begin{aligned}
\sigma^{2} & =(1-\epsilon) \sigma_{q}^{2}+\epsilon \mathbb{E} \sigma_{\overline{\mathbf{h}} \neq \overline{\mathbf{h}}}^{2}(\mathbf{h}, \hat{\mathbf{h}}) \\
& \leq(1-\epsilon) \sigma_{q}^{2}+\epsilon \\
& \leq \sigma_{q}^{2}+\epsilon,
\end{aligned}
$$

where $\sigma_{q}^{2}$ represents the mean-square quantization error and $\mathbb{E} \sigma_{\overline{\mathbf{h}} \neq \overline{\hat{\mathbf{h}}}}^{2}(\mathbf{h}, \hat{\mathbf{h}})$ represents the mean-square CDI error when feedback error occurs. The first inequality is obtained as $\mathbb{E} \sigma_{\overline{\mathbf{h}} \neq \overline{\hat{\mathbf{h}}}}^{2}(\mathbf{h}, \hat{\mathbf{h}})$ is upper-bounded by 1 and the second inequality is obtained by adding $\epsilon \sigma_{q}^{2}$.

We select this upper bound of MSE of CSIT as the performance metric and minimize it following the constraints on the total feedback interval and user's power constraint, obtaining the optimal values for $T_{a}, T_{q}$ and $b$ (the number of bits/symbol - this parameter governs the constellation size and hence quantization error). The results thus obtained show the superiority of this hybrid approach over the classical training based only CSIT.

For RVQ, the exact expression for the mean-square quantization error $\sigma_{q}^{2}$ has been given in [15], [12] but we'll use a very simple and tight upper bound from [12]

$$
\sigma_{q}^{2} \leq 2^{\frac{-B}{M-1}}
$$

Putting the value of $\sigma_{q}^{2}$ using $B=b T_{q}$ in eq. (14), we get the upper bound of MSE of CSIT as

$$
\sigma^{2} \leq 2^{\frac{-b T_{q}}{M-1}}+\epsilon
$$

This equation shows us the basic trade-off involved. If a large outage rate $b$ is used in the system, it will allow the user to select a larger codebook (with $2^{B}$ code words where $B=b T_{q}$ ) and hence quantization error will be negligible. But the choice of larger outage rate will plague the final CSIT estimation error by introducing a lot many outages as for a lot of realizations, BS won't be able to decode the feedback correctly. On the other hand, if a very pessimistic outage rate $b$ is selected for transmission corresponding to very bad channel conditions, the degradation due to outage probability will fade away. But there will be fewer codewords in the codebook and, in this case, CSIT estimate at the BS will be of poor quality due to large quantization error.

\section{B. Training and Feedback Resource Split Optimization}

Pilot sequence transmission from the user to the BS for an interval of length $T_{a}$, given in eq. (8), can be equivalently written in a simplified form as

$$
\mathbf{y}_{\mathbf{a}}=\sqrt{P T_{a}} \mathbf{h}+\mathbf{n}_{\mathbf{a}}
$$

where $P$ is the user's power constraint and $\mathbf{y}_{\mathbf{a}}, \mathbf{h}, \mathbf{n}_{\mathbf{a}}$ are the received signal, the channel vector and the noise respectively, all column vectors of dimension $M$. BS can make MMSE estimate $\hat{\mathbf{h}}_{\mathbf{a}}$ of the channel $\mathbf{h}$ as

$$
\hat{\mathbf{h}}_{\mathbf{a}}=\frac{\sqrt{P T_{a}}}{P T_{a}+1} \mathbf{y}_{\mathbf{a}} .
$$

As channel entries are i.i.d. standard Gaussian, MMSE estimation error $\tilde{\mathbf{h}}_{\mathbf{a}}=\mathbf{h}-\hat{\mathbf{h}}_{\mathbf{a}}$ has also i.i.d. Gaussian distributed entries as $\tilde{\mathbf{h}}_{\mathbf{a}} \sim \mathcal{C N}\left(\mathbf{0}, \sigma_{a}^{2} \mathbf{I}_{\mathbf{M}}\right)$, where $\mathbf{I}_{\mathbf{M}}$ represents the $M$-dimensional identity matrix and the MSE per channel coefficient $\sigma_{a}^{2}$ is given by

$$
\sigma_{a}^{2}=\frac{1}{P T_{a}+1}
$$

Similarly the estimate $\hat{\mathbf{h}}_{\mathbf{a}}$ has i.i.d. Gaussian entries and is distributed as $\hat{\mathbf{h}}_{\mathbf{a}} \sim \mathcal{C N}\left(\mathbf{0}, \frac{P T_{a}}{P T_{a}+1} \mathbf{I}_{\mathbf{M}}\right)$.

Now we focus our attention on the digital feedback interval of the CSIT acquisition, given in eq. (10). The signal received during one symbol interval of this phase is given by

$$
\mathbf{y}_{\mathbf{q}}=\sqrt{P} \mathbf{h} x_{q}+\mathbf{n}_{\mathbf{q}}
$$


where $x_{q}$ represents the scalar feedback symbol transmitted by the user and $\mathbf{y}_{\mathbf{q}}, \mathbf{h}, \mathbf{n}_{\mathbf{q}}$ are $M$-dimensional column vectors representing respectively the observed signal, channel and noise for this particular symbol interval. To decode this information, BS uses the estimate $\hat{\mathbf{h}}_{\mathbf{a}}$ that it developed during the training phase. So the above equation can be written as

$$
\mathbf{y}_{\mathbf{q}}=\sqrt{P} \hat{\mathbf{h}}_{\mathbf{a}} x_{q}+\sqrt{P} \tilde{\mathbf{h}}_{\mathbf{a}} x_{q}+\mathbf{n}_{\mathbf{q}} .
$$

Average signal-to-noise-ratio (SNR) at the BS with channel $\hat{\mathbf{h}}_{\mathbf{a}}$ known can be computed to be

$$
\operatorname{SNR}\left(\hat{\mathbf{h}}_{\mathbf{a}}\right)=\frac{P\left\|\hat{\mathbf{h}}_{\mathbf{a}}\right\|^{2}}{P \sigma_{a}^{2}+1} .
$$

Putting the value of $\sigma_{a}^{2}$, SNR will become

$$
\operatorname{SNR}\left(\hat{\mathbf{h}}_{\mathbf{a}}\right)=\frac{P\left\|\hat{\mathbf{h}}_{\mathbf{a}}\right\|^{2}}{\frac{P}{P T_{a}+1}+1} .
$$

We can do the magnitude scaling of the estimated channel vector $\hat{\mathbf{h}}_{\mathbf{a}}$ so as to convert $\left\|\hat{\mathbf{h}}_{\mathbf{a}}\right\|^{2}$ in a standard chi-square random variable having $2 M$ degrees of freedom (DOF), denoted as $\chi_{2 M}^{2}$. So the SNR becomes

$$
\operatorname{SNR}\left(\chi_{2 M}^{2}\right)=\frac{P^{2} T_{a}}{2\left(P+P T_{a}+1\right)} \chi_{2 M}^{2} .
$$

This equation has been obtained by dividing and multiplying the right hand side of the preceding equation by $\frac{P T_{a}}{2\left(P T_{a}+1\right)}$ as $\frac{2\left(P T_{a}+1\right)}{P T_{a}}\left\|\hat{\mathbf{h}}_{\mathbf{a}}\right\|^{2}$ actually represents the sum of squares of $2 M$ real independent zero-mean unit-variance Gaussian variables which is a standard chi-square random variable with $2 M$ DOF.

The outage probability, $\epsilon$, of this channel corresponding to the outage rate $b$, can be written as

$$
\begin{aligned}
\epsilon & =\mathbb{P}\left[\log \left(1+\operatorname{SNR}\left(\chi_{2 M}^{2}\right)\right) \leq b\right] \\
& =\mathbb{P}\left[\log \left(1+\frac{P^{2} T_{a}}{2\left(P+P T_{a}+1\right)} \chi_{2 M}^{2}\right) \leq b\right],
\end{aligned}
$$

where $\mathbb{P}$ denotes the probability of an event. This relation can be inverted to obtain the outage rate $b$ corresponding to the outage probability $\epsilon$, as given below

$$
b=\log \left(1+\frac{P^{2} T_{a}}{2\left(P+P T_{a}+1\right)} F^{-1}(\epsilon)\right),
$$

where $F^{-1}($.$) is the inverse of the standard cumulative distri-$ bution function (CDF) of $\chi_{2 M}^{2}$ distributed variable.

Finding the above expression, which relates the outage rate of digital feedback with the training length $T_{a}$, we can put formally our optimization problem (the minimization of the mean-square CSIT error given in eq. (16)) as follows:

$$
\min _{T_{a}, b}\left[2^{\frac{-b\left(T_{f b}-T_{a}\right)}{M-1}}+\epsilon\right]
$$

where we have used $T_{f b}=T_{a}+T_{q}$ and, $b$ and $\epsilon$ are related through eq. (25) and eq. (26). The constraints for this minimization are:

$$
\begin{aligned}
T_{a} & \geq 1 \quad, \quad T_{a} \leq T_{f b} \\
b & \geq 0 \quad .
\end{aligned}
$$

Apart from these, the user has to take care of its UL power constraint. The analytical solution to the above optimization problem does not bear closed form expression but its numerical optimization is quite trivial.

\section{Simulation RESUlts}

Our simulation environment consists of a BS with $M=4$ antennas and a single user with a single antenna. The channel model is same as described in Section II. The feedback interval $T_{f b}$ is fixed to 20 symbol intervals for all simulations.

\section{A. Optimization Results for Continuous Constellations}

Here we present the results when outage rate $b$ is not constrained to be an integer and can assume any positive real value. The optimization of the objective function, given in section $\mathrm{V}$ gives us the values for the optimal training length $T_{a}$ and the optimal outage rate $b$ for various values of user's power constraint, which is equal to the UL SNR as the noise at every BS antenna has been normalized to have unit variance. We have plotted the optimal values of training length $T_{a}$, corresponding values of quantized feedback interval $T_{q}$ and the outage rate $b$ in Fig. (2).

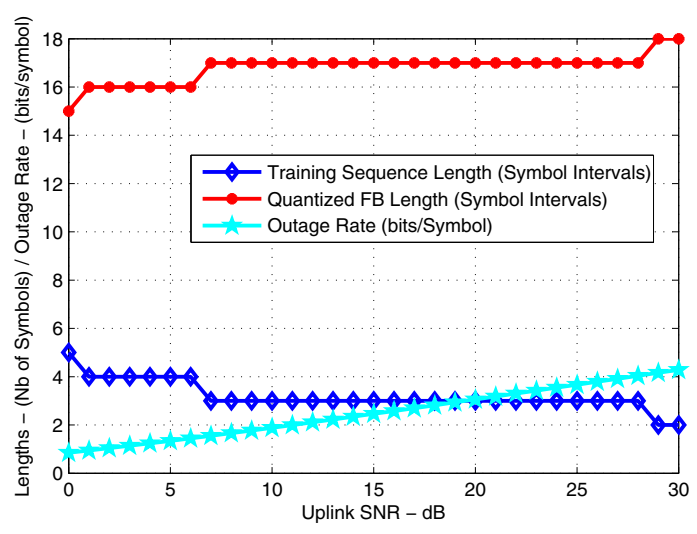

Fig. 2. Optimal Lengths and Outage Rate: $T_{f b}=20$ and $M=4$. With increase in SNR, both the length of quantized feedback interval $T_{q}$ and the outage rate $b$ increase gradually.

Knowing the values of $\epsilon$ and $T_{q}$, computed based upon the optimal values of $T_{a}$ and $b$, allows us to compute the upper bound of the final CSIT error eq. (16). These values have been plotted in dB scale in Fig. 3. For comparison purpose, we have also plotted the CSIT error when classical training spans the full interval reserved for CSIT acquisition. This plot clearly shows the interest of our hybrid two-staged CSIT acquisition strategy as, from medium to large SNR values, CSIT error incurred by this scheme is much less than the error obtained by training based only CSIT acquisition.

At low SNR values, this two stage scheme performs worse than the training based traditional scheme. This happens because we have restricted our final estimate to come from the digital feedback. Here total feedback resource (SNR and $T_{f b}$ ) does not allow transmission of sufficient number of bits through the channel so quantization error is quite large. 


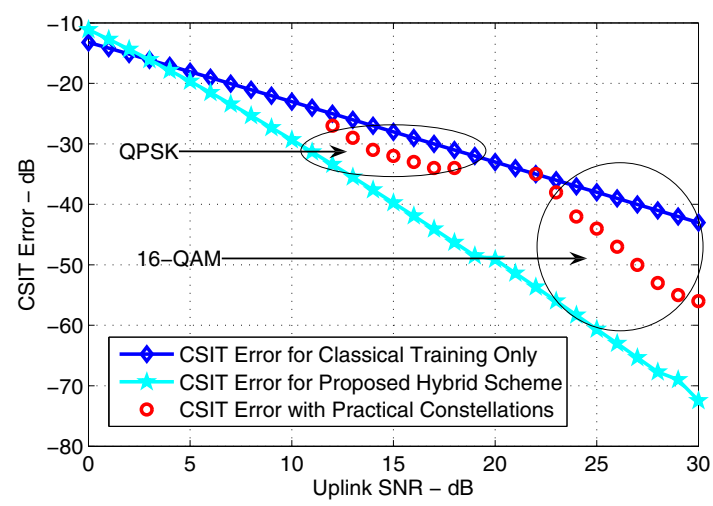

Fig. 3. Mean-Square CSIT Errors: $T_{f b}=20$ and $M=4$. Novel hybrid scheme performs much better than classical training based CSIT acquisition. Gains are significant even with naive use of practical constellations without any coding.

This gets aggravated due to poor training based estimate based upon which these bits are decoded, further degrading the performance. This degradation can be easily avoided by selecting an SNR threshold below which traditional training based scheme is employed.

\section{B. Transmission with Practical Constellations}

Although the plots show the potential of our hybrid approach over the classical training only scheme, with gains which are increasing in SNR, yet these results have been obtained using the real positive values for $b$. Constellations, used in practice, have number of points which are an integer power of 2. This practical limitation dictates that the $\epsilon$-outage rate $b$ should be an integer. The MSE of CSIT, obtained by transmission of QPSK and 16-QAM symbols, has been plotted for certain SNR values. We remark that these constellations incur performance degradation as compared to the theoretical analysis. The reason is analysis might show that $b=2.7$, in this case if we select smaller constellation i.e., QPSK $(b=2)$, we actually let the quantization error increase, if we select e.g. 16-QAM $(b=4)$, it will cause the outage probability to increase. In any case, increase in either the outage probability or the quantization error will cause to degrade the quality of the CSIT estimate at the BS.

This performance degradation can be avoided by selecting a larger constellation (higher $b$ ) but doing coding with the help of extra bits i.e., codebook size is not increased. In this way, all extra bits are used as parity bits and help combat the channel noise and outages. This point is the focus of ongoing research and results will be presented elsewhere.

\section{CONCLUSIONS}

We presented a novel approach of acquiring CSIT at the BS for the DL transmission in a reciprocal TDD MIMO communication system. Traditional CSIT acquisition in TDD systems relies on the use of training sequences in an UL slot. This approach fails to fully exploit the channel reciprocity by ignoring the shared knowledge of an identical channel between the BS and the user. Instead, we propose a strategy combining the use of a training sequence together with a limited feedback channel. For the transmission of the quantized feedback, ergodic rates are far beyond the reality due to stringent delay constraints. We characterize the optimal CSIT acquisition setup and give an outage-rate based approach which allows the optimum partitioning of resource between the training and the quantized feedback. Superior performance is demonstrated due to better exploitation of the reciprocity principle and CSIT quality improves significantly.

\section{ACKNOWLEDGMENTS}

Eurecom's research is partially supported by its industrial members: BMW Group, Bouygues Telecom, Cisco, Hitachi, ORANGE, SFR, Sharp, STMicroelectronics, Swisscom, Thales. The research work leading to this paper has also been partially supported by the European Commission through the FP7 ICT Network of Excellence NewCom++ and project WHERE, and by the French ANR project APOGEE.

\section{REFERENCES}

[1] I. E. Telatar, "Capacity of multi-antenna Gaussian channels," European Transactions on Telecommunications, pp. 585-595, November 1999.

[2] G. J. Foschini and M. J. Gans, "On limits of wireless communications in a fading environment when using multiple antennas," Wireless Personal Communications, vol. 6, pp. 311-335, 1998.

[3] G. Caire and S. Shamai (Shitz), "On the achievable throughput of a multiantenna Gaussian broadcast channel," IEEE Trans. on Information Theory, vol. 49, pp. 1691-1706, July 2003.

[4] P. Viswanath and D. Tse, "Sum capacity of the multiple antenna Gaussian broadcast channel and uplink-downlink duality," IEEE Trans. on Information Theory, vol. 49, pp. 1912-1921, August 2003.

[5] D. Gesbert, M. Kountouris, J. R. W. Heath, C. B. Chae, and T. Salzer, "From single user to multiuser communications: Shifting the MIMO paradigm,” IEEE Sig. Proc. Magazine, 2007.

[6] P. Viswanath, D. N. C. Tse, and R. Laroia, "Opportunistic beamforming using dumb antennas," IEEE Transactions on Information Theory, vol. 48, pp. 1277-1294, June 2002.

[7] M. Sharif and B. Hassibi, "On the capacity of MIMO broadcast channels with partial side information," IEEE Transactions on Information Theory, vol. 51, pp. 506-522, February 2005.

[8] T. L. Marzetta, "How much training is required for multiuser MIMO?," in Proc. Asilomar Conference on Signals, Systems and Computers, Pacific Grove, CA, USA, November 2006, pp. 359-363.

[9] T. Marzetta and B. Hochwald, "Fast transfer of channel state information in wireless systems," IEEE Trans. on Signal Processing, vol. 54, pp. 1268-1278, April 2006.

[10] U. Salim and D. Slock, "Broadcast channel: Degrees of freedom with no CSIR," in Proc. Allerton Conf. on Communication, Control, and Computing, 2008.

[11] D. J. Love, R. W. Heath Jr., W. Santipach, and M. L. Honig, "What is the value of limited feedback for MIMO channels?," IEEE Communications Magazine, vol. 42, pp. 54-59, October 2004.

[12] N. Jindal, "MIMO broadcast channels with finite rate feedback," IEEE Trans. on Information Theory, vol. 52, pp. 5045-5060, November 2006.

[13] T. Yoo, N. Jindal, and A. Goldsmith, "Multi-antenna downlink channels with limited feedback and user selection," IEEE Journal on Selected Areas in Communications, vol. 24, September 2007.

[14] T. Marzetta and B. Hochwald, "Capacity of a mobile multipleantenna communications link in Rayleigh flat fading," IEEE Trans. on Information Theory, vol. 45, pp. 139-157, January 1999.

[15] C. Au-Yeung and D. J. Love, "On the performance of random vector quantization limited feedback beamforming in a MISO system," IEEE Trans. on Wireless Communications, vol. 6, pp. 458-462, February 2007.

[16] D. Tse and P. Viswanath, Fundamentals of Wireless Communications, Cambridge, U.K. Cambridge Univ. Press, 2005. 